\title{
Modelos de Markov Aplicados na Avaliação de Usabilidade de um Sistema para Educação à Distância
}

\author{
Janaina R Penedo ${ }^{1}$, Morganna Diniz ${ }^{1}$, Simone Bacellar Leal Ferreira ${ }^{1}$, Denis S. \\ Silveira $^{2}$, Eliane Capra ${ }^{1}$ \\ ${ }^{1}$ Universidade Federal do Estado do Rio de Janeiro (UNIRIO) \\ Av. Pasteur, 296 - Urca - CEP 22290-240 - Rio de Janeiro - RJ - Brazil
}

\author{
${ }^{2}$ Universidade Federal de Pernambuco (UFPE) \\ Av. dos Funcionários, s/n - Recife - PE - Brazil \\ \{janaina.penedo, morganna, simone, eliane.capra\}@uniriotec.br, \\ dsilveira@ufpe.br
}

\begin{abstract}
With the growth of web-based systems, usability has gained visibility in the Human-Computer Interaction area (HCI). Usability evaluation methods consist of systematic processes of data collection with the purpose of analyzing how users handle a product in order to execute tasks in a computer environment. The present research aims at analyzing the usability of a remote learning system using a quantitative usability evaluation method through Markov models. These models allow a series of measures of interest to be calculated and they have been successfully utilized in the evaluation of computing and communication systems, but they have not been much used in the evaluation of system usability
\end{abstract}

Resumo. A usabilidade ganhou destaque na área de Interação HumanoComputador (IHC). Os métodos de avaliação de usabilidade consistem de processos de coleta de dados com a finalidade de analisar como os usuários usam um produto para executar suas tarefas. A presente pesquisa tem por objetivo avaliar a usabilidade de um sistema de educação à distância utilizando uma avaliação de usabilidade quantitativa através de modelos markovianos a fim de identificar falhas de usabilidade na interface. Esses modelos permitem o cálculo de uma série de medidas de interesse e têm sido utilizados com sucesso na avaliação de sistemas, mas são ainda pouco aproveitados em avaliações de usabilidade de sistemas Web.

\section{Introdução}

O advento dos sistemas de informação para ambiente Web, incluindo os de Educação à Distância (EAD), difundiu a importância para atenção à usabilidade [Preece et al. 2005], característica que determina se o manuseio de um produto é fácil e rapidamente aprendido, dificilmente esquecido, não provoca erros operacionais, oferece um alto grau de satisfação para seus usuários, e eficientemente resolve as tarefas para as quais ele foi projetado [Ferreira e Nunes 2008], [Nielsen e Loranger 2007], [Rocha e Baranauska 2000]. 
Uma das formas de prover essa usabilidade é por meio da avaliação de interfaces, onde é possível detectar problemas no uso do sistema. Para verificar a usabilidade existem métodos de avaliação, que podem ser empíricos (os avaliadores envolvem usuários para a coleta de dados) ou analíticos (os analisadores examinam aspectos de uma interface, sem a participação de usuários). Entre os processos de avaliação analítica encontra-se a inspeção baseada em modelos, ainda pouco explorada. Portanto, a presente pesquisa, de caráter quantitativo e exploratório, tem por objetivo avaliar a usabilidade em sistemas de informação com base em modelos matemáticos, com a finalidade de apresentar uma forma de inspeção simples que permita identificar falhas de usabilidade na interface. O benefício do modelo proposto é a possibilidade de se avaliar a usabilidade de uma interface tanto em fase inicial (pré-projeto) quanto em fase de projeto.

O modelo aqui proposto foi avaliado por meio da condução de estudo de caso controlado. Espera-se que essa contribuição, embora inicialmente focada em sistemas EAD, possa ser estendida a outros sistemas, de modo a auxiliar na construção e adaptação de interfaces mais aderentes às recomendações de usabilidade de sistemas.

\section{Avaliação de Usabilidade}

A avaliação de interfaces consiste de um processo sistemático de coleta de dados com a finalidade de analisar como os usuários usam um produto para executar suas tarefas em algum ambiente computacional [Preece et al. 2005]. Dentre seus principais objetivos destacam-se: avaliar a qualidade de um projeto de interface, identificar possíveis problemas de interação, verificar seus requisitos de usabilidade, entre outros [Prates e Barbosa 2003].

Em relação à maneira de coletar dados, o método pode variar segundo o propósito da avaliação. Quando a avaliação de uma interface é feita com a presença dos usuários, os processos recebem o nome de "métodos de observação" ou "teste com usuários". Quando a avaliação é feita sem a participação dos usuários, são chamados de "métodos de inspeção" ou "métodos analíticos ou prognósticos" [Barbosa e Silva 2010], [Prates e Barbosa 2003].

Nos métodos analíticos, a análise é realizada por avaliadores ou especialistas, que inspecionam ou examinam aspectos de uma interface. Em geral, esses métodos são usados para identificar e analisar os problemas de usabilidade com a finalidade de gerar recomendações para consertá-los e melhorar a qualidade da interface [Prates e Barbosa 2003]. Existem diversos tipos de avaliações analíticas, como avaliação heurística, revisão de guidelines (guias de referência), inspeções de consistência, percurso cognitivo, percurso pluralista, inspeção baseada em padrões, inspeção baseada em taxonomia e inspeção baseada nos modelos markovianos.

Para realizar as avaliações são necessárias algumas técnicas. A partir de um estudo qualitativo e comparativo dos métodos utilizados para avaliação da usabilidade de autores conceituados da área de IHC, como Shackel [Shackel 1986], Bastien e Scapin [Bastien e Scapin 1993], Jordan [Jordan 1998], Shneiderman [Shneiderman 1998], Quesenbery [Quesenberry 2001], Gassenferth et al [Gassenferth et al 2008], propuseram um conjunto de regras para medir o grau de usabilidade de sistemas, que deram origem a seis critérios convergentes para avaliação da usabilidade, são eles: 
Facilidade de aprender: estabelece que o sistema não somente deve ser fácil de aprender e manusear, mas também deve reagir ao contexto do uso e ser claro na apresentação das expressões para manter uma boa interação entre usuário e sistema, facilitando seu aprendizado;

Facilidade de relembrar: determina quais ações realizadas no sistema devem ser facilmente relembradas, mesmo quando executadas esporadicamente ou depois de longos intervalos;

Controle de Erros: estabelece que o usuário deve ser claramente informado sobre o que ocasionou o erro de modo a resolvê-lo facilmente.

Eficiência: o usuário deve poder operar o sistema em alto grau de produtividade e desempenho na realização das tarefas e de forma rápida;

Eficácia: o sistema deve fazer a coisa certa e da melhor forma possível. O sistema deve executar a tarefa com um mínimo de passos, ou seja, proporcionar menos ações do usuário para concluir uma determinada tarefa;

Satisfação: diz respeito à percepção do usuário sobre o uso do sistema. Está ligado à opinião do usuário com relação às características do sistema como satisfação e conforto no uso.

\section{Modelos Markovianos aplicados na avaliação da Usabilidade}

A utilização de Modelos de Markov para a avaliação da usabilidade foi proposta em 2001, por Thimbleby, Cairns e Jones [Thimbleby et al 2001]. Os autores utilizaram essa técnica para propor uma ferramenta para a avaliação de usabilidade em dispositivos como forno de microondas e telefones celulares, antes da geração de um protótipo. Para os autores, a usabilidade pode ser avaliada de acordo com a premissa que define se um dispositivo é fácil ou difícil de utilizar. Após o estudo, foi apresentada uma ferramenta que pode ser utilizada por projetistas para avaliação da usabilidade através de um gráfico de custo/conhecimento, não sendo necessários grandes conhecimentos matemáticos [Thimbleby et al 2001].

Em 2005, Kitajima et al [Kitajima et al 2005] propuseram um método para avaliar quantitativamente a usabilidade da Enciclopédia on-line Encarta. Para isso, foi utilizado o método de inspeção de usabilidade conhecido como "Cognitive Walkthrough" (Percurso Cognitivo) e cadeias de Markov. Os resultados mostraram que a utilização de cadeias de Markov pode fornecer resultados simples e rápidos, auxiliando a utilização desse método de inspeção.

Os estudos realizados em [Kitajima et al 2005] e [Thimbleby et al 2001] apontaram que a vantagem em realizar inspeção de usabilidade baseada em modelos markovianos é a possibilidade de se obter métricas que forneçam comparações quantitativas para uma rápida avaliação da usabilidade de um sistema.

A proposta deste trabalho é dar continuidade as propostas de [Kitajima et al 2005] e [Thimbleby et al 2001] avaliando a usabilidade de um sistema de EAD através de modelos de markov, porém, utilizando-se as regras definidas por Gassenferth et al [Gassenferth et al 2008]. 
Considerando que um usuário tem escolhas em cada operação, é importante levar em conta a quantidade de maneiras possíveis que um determinado objetivo pode ser alcançado. O número de estados/transições que o usuário necessita para alcançar seu objetivo é uma forma simples de medir as dificuldades encontradas em um sistema de informação [Thimbleby et al 2001].

Um sistema evolui através das transições em seus estados [Booch 1994]. Assim, os modelos markovianos podem ser utilizados para representar mudanças ou transições entre estados quando o estado anterior é irrelevante para a predição dos estados seguintes [Kitajima et al 2005]. Considerando que as ações, dependendo das suas respectivas condições, podem indicar uma mudança de estado, torna-se necessário definir as probabilidades que indicam essas mudanças (transições) [Kleinrock 1975].Em uma cadeia de Markov, o símbolo $p_{i j}$ é usado para representar a probabilidade de que, dado que o sistema está no estado $i$ em certo momento, ele passe para o estado $j$ no intervalo de tempo seguinte. Portanto, $p_{i j}$ representa as probabilidades de transição da cadeia de Markov. Para completar o modelo é necessário fornecer uma distribuição de probabilidades para o estado inicial, ou seja, definir as probabilidades iniciais de cada estado do sistema (início da observação). $\mathrm{O}$ vetor $\pi(0)$, que representa esta distribuição, é definido pela Equação 1, onde $\pi_{\mathrm{i}}(0)$ representa a probabilidade do sistema estar no estado $i$ antes de qualquer transição no sistema.

$\pi(0)=\left[\pi_{0}(0), \pi_{1}(0), \pi_{2}(0), \ldots\right]$

Considerando que $P$ é a matriz de probabilidades de transição entre os estados do sistema, pode-se calcular as probabilidades dos estados após $n$ transições, representado pela Equação 2 (Kleinrock, 1975).

$\pi(\mathrm{n})=\pi(0) \mathrm{P}^{\mathrm{n}}$

Quando a cadeia é irredutível (todos os estados se comunicam entre si) e $\mathrm{n} \rightarrow \infty$, tem-se $\pi$, que é o vetor das probabilidades estacionárias de uma cadeia irredutível, dada pela Equação 3:

$\pi=\pi \mathrm{P}^{\mathrm{n}}$

Isto significa que, após um período muito longo de observação, espera-se encontrar o sistema no estado $i$ com probabilidade $\pi_{i}$. Porém, ainda convém fazer duas observações quanto ao uso de Markov para avaliação de usabilidade: (a) sobre o cálculo das probabilidades de transição, que podem ser calculadas a partir da observação das transições de estado em um sistema real, ou seja, pode-se considerar, por exemplo, as interfaces de um sistema como os estados de uma cadeia de Markov e as diferentes formas de navegação entre as páginas como probabilidades de transição; (b) sobre a escolha de critérios para analisar a usabilidade de um sistema representado por uma cadeia de Markov.

Embora os modelos markovianos sejam utilizados em uma infinidade de áreas científicas [Cunha, Machado, Figueiredo Filho e Sanquetta 2010; Marinho, Cardoso e Almeida 2010; Morais, Bertoldi e Anjos 2010], não existem ainda critérios definidos na literatura para a avaliação de usabilidade a partir de uma cadeia de Markov. Logo, para este trabalho optou-se pela utilização dos critérios de Gassenferth et al [Gassenferth et al 2008] pelo fato deles terem se baseado em um estudo qualitativo e comparativo dos 
métodos utilizados para avaliação da usabilidade de autores conceituados da área de IHC, apresentados na Seção 2.

\section{Método de Pesquisa}

A pesquisa de caráter quantitativo e exploratório foi realizada com base em um estudo de caso. Esse estudo se deu em cinco etapas, descritas a seguir:

Coleta e análise de dados: nessa fase foram coletados e analisados os dados referentes aos acessos dos usuários de um sistema de educação à distância. Após a definição dos dados necessários, foi gerado o $\log$ com as informações referentes a três meses do curso de graduação em computação oferecido no sistema selecionado para objeto de estudo.

Identificação das possíveis funções do sistema: nessa fase foram levantadas e identificadas as funções que o sistema disponibiliza aos alunos, onde uma função corresponde a um conjunto de facilidades/serviços do ambiente de EAD.

Transformação das funções do sistema em estados de Markov: depois de identificadas as funções, as mesmas foram transformadas em estados seguindo a definição de modelos markovianos.

Geração da matriz de probabilidades entre os estados: depois de definidos os estados, foi gerada a matriz de probabilidades de transição de estados. Esta matriz representa as probabilidades de navegação entre as páginas web do site mapeadas a partir do estudo do log do sistema de EAD.

Análise dos resultados: os critérios de Gassenferth et al (2008) foram então usados para avaliar a usabilidade do site.

\section{Avaliação}

Para a realização desta pesquisa foi realizado um estudo de caso controlado, tendo como objeto o sistema disponibilizado pelo CEDERJ [Cederj 2011] para o acesso dos alunos do Curso Superior de Tecnologia de Sistemas de Computação referentes aos meses de agosto, setembro e outubro de 2010.

O Consórcio CEDERJ da Fundação CECIERJ reúne seis universidades públicas sediadas no estado do Rio de Janeiro.

O aluno do Consórcio CEDERJ está matriculado em uma das universidades do consórcio, dependendo do curso e do pólo regional a que esteja vinculado.

O formato das aulas gravadas é o mesmo encontrado nos cursos presenciais: o aluno visualiza o professor e as transparências do tópico em discussão e pode interagir como se estivesse vendo um filme em DVD, podendo parar, avançar, voltar ou ir direto a uma parte da apresentação. Além disso, o aluno pode ser convidado, durante a aula, a realizar alguma tarefa no próprio computador com o objetivo de consolidar a matéria em estudo.

A primeira etapa da pesquisa coletou e analisou os dados para o cálculo das métricas como: tempo médio dos usuários conectados à plataforma, distribuição dos acessos dos alunos ao sistema nas 24 horas do dia e nos sete dias da semana, os serviços 
mais solicitados ao sistema, caminho percorrido dentro do sistema, dentre outros. Este estudo mostrou que existe uma similaridade no comportamento dos alunos do curso de computação. Um exemplo desta afirmativa pode ser visto na Figura 1 que apresenta $o$ tempo médio dos usuários no sistema.

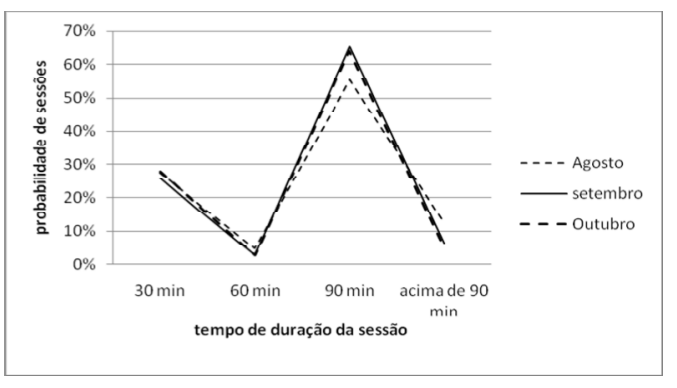

Figura 1. Tempo médio dos usuários na Plataforma CEDERJ

Devido à similaridade nos comportamentos dos usuários nos meses analisados e, por questão de espaço, este trabalho apresenta a análise de apenas um mês de acesso ao sistema de EAD do CEDERJ. O mês escolhido foi agosto por apresentar o maior número de requisições de serviço e de usuários ativos. Entendem-se como usuários ativos no sistema todos aqueles que se conectaram na plataforma no período analisado; e sessão como o tempo correspondente ao intervalo de uma ação de login e uma ação de logout de um determinado usuário. A Tabela 1 apresenta as diversas métricas usadas neste trabalho para caracterizar o comportamento dos alunos quando acessam o sistema.

Tabela 1. Métricas Utilizadas na Pesquisa.

\begin{tabular}{|l|c|c|c|c|}
\hline & Agosto & Setembro & Outubro & Novembro \\
\hline Usuários Ativos & 1.956 & 1.545 & 1.484 & 1473 \\
\hline Sessões Ativas & 20.935 & 21.434 & 14.681 & 15.580 \\
\hline Requisições Recebidas & 375.959 & 194.801 & 140.187 & 151.449 \\
\hline Média de Tempo Conectado & $00: 58: 50$ & $00: 53: 00$ & $01: 04: 55$ & $00: 50: 15$ \\
\hline
\end{tabular}

$\mathrm{Na}$ segunda etapa da pesquisa foram identificadas as funções ou ferramentas que a plataforma disponibiliza aos alunos. A plataforma CEDERJ disponibiliza atualmente 81 módulos diferentes de serviço para os seus usuários. A partir da avaliação dos $\log s$, é possível observar que as solicitações dos usuários se concentram em onze módulos. A descrição dos módulos mais utilizados pelos alunos pode ser vista na Tabela 2.

Tabela 2. Funções mais utilizadas

\begin{tabular}{|c|l|l|}
\hline \multicolumn{2}{|c|}{ Módulo } & \multicolumn{2}{|c|}{ Ações Disponíveis } \\
\hline $\mathbf{1}$ & Login & $\begin{array}{l}\text { Identificação do usuário e do ambiente a ser } \\
\text { estudado. }\end{array}$ \\
\hline $\mathbf{2}$ & Fórum & $\begin{array}{l}\text { Discussão de um determinado assunto pelos } \\
\text { alunos, tutores, professores e coordenadores do } \\
\text { curso. }\end{array}$ \\
\hline $\mathbf{3}$ & Sala de tutorial & Discussão das disciplinas com os tutores. \\
\hline $\mathbf{4}$ & $\begin{array}{l}\text { Gerenciador de } \\
\text { arquivos }\end{array}$ & Upload/Download de arquivos. \\
\hline $\mathbf{5}$ & Aviso & Visualização de mensagens do CEDERJ. \\
\hline $\mathbf{6}$ & E-Mail & Acesso e utilização de e-mails pela plataforma. \\
\hline $\mathbf{7}$ & $\begin{array}{l}\text { Grade de de } \\
\text { disciplina }\end{array}$ & Acesso as disciplinas do curso. \\
\hline
\end{tabular}




\begin{tabular}{|c|l|l|}
\hline $\mathbf{8}$ & Mural do curso & $\begin{array}{l}\text { Visualização de mensagens da coordenação do } \\
\text { curso. }\end{array}$ \\
\hline $\mathbf{9}$ & Mural dos pólos & Visualização de mensagens do pólo. \\
\hline $\mathbf{1 0}$ & $\begin{array}{l}\text { Sala de } \\
\text { disciplina }\end{array}$ & $\begin{array}{l}\text { Acesso a todo ferramental alocado para as } \\
\text { disciplinas. }\end{array}$ \\
\hline $\mathbf{1 1}$ & $\begin{array}{l}\text { Material } \\
\text { didático }\end{array}$ & $\begin{array}{l}\text { Acesso ao material complementar disponível para } \\
\text { as disciplinas. }\end{array}$ \\
\hline
\end{tabular}

A terceira etapa desta pesquisa consistiu na transformação das funções em estados de uma cadeia de Markov. A modelagem através de Markov deve representar um conjunto do modelo em estudo, onde apenas o período de utilização do sistema é considerado, ignorando assim o período em que o usuário não está conectado (logado) no sistema. Isto é representado no modelo pelo agrupamento dos estados de entrada e saída do sistema em um único estado. O quadro da Figura 2 apresenta a matriz de probabilidades de transições do sistema referentes ao mês de agosto/2010.

\begin{tabular}{|r|r|r|r|r|r|r|r|r|r|r|r|}
\hline & \multicolumn{1}{|c|}{1} & \multicolumn{1}{c|}{$\mathbf{2}$} & \multicolumn{1}{c|}{3} & \multicolumn{1}{c|}{4} & \multicolumn{1}{c|}{5} & \multicolumn{1}{c|}{6} & \multicolumn{1}{c|}{7} & \multicolumn{1}{c|}{8} & \multicolumn{1}{c|}{9} & \multicolumn{1}{c|}{10} & \multicolumn{1}{c|}{11} \\
\hline $\mathbf{1}$ & 0,2 & 0,01 & 0,18 & 0,02 & 0,02 & 0,06 & 0,18 & 0,01 & 0,01 & 0,29 & 0,02 \\
\hline $\mathbf{2}$ & 0,16 & 0,13 & 0,2 & 0,02 & 0,02 & 0,05 & 0,18 & 0,01 & 0,01 & 0,2 & 0,02 \\
\hline 3 & 0,17 & 0,01 & 0,34 & 0,02 & 0,02 & 0,05 & 0,17 & 0,01 & 0,01 & 0,19 & 0,01 \\
\hline 4 & 0,15 & 0,01 & 0,12 & 0,32 & 0,02 & 0,04 & 0,14 & 0,01 & 0,01 & 0,17 & 0,01 \\
\hline $\mathbf{5}$ & 0,16 & 0,01 & 0,13 & 0,2 & 0,11 & 0,03 & 0,15 & 0,01 & 0,01 & 0,18 & 0,01 \\
\hline 6 & 0,1 & 0 & 0,07 & 0,13 & 0,01 & 0,51 & 0,08 & 0 & 0,01 & 0,09 & 0 \\
\hline 7 & 0,12 & 0,01 & 0,12 & 0,28 & 0,02 & 0,04 & 0,2 & 0,01 & 0,01 & 0,18 & 0,01 \\
\hline 8 & 0,15 & 0,01 & 0,11 & 0,21 & 0,02 & 0,05 & 0,13 & 0,02 & 0,12 & 0,17 & 0,01 \\
\hline 9 & 0,16 & 0,01 & 0,13 & 0,21 & 0,02 & 0,05 & 0,13 & 0,08 & 0,02 & 0,18 & 0,01 \\
\hline 10 & 0,13 & 0,01 & 0,14 & 0,24 & 0,03 & 0,04 & 0,18 & 0,01 & 0,01 & 0,2 & 0,01 \\
\hline 11 & 0,14 & 0 & 0,11 & 0,24 & 0,02 & 0,04 & 0,19 & 0,01 & 0,01 & 0,18 & 0,06 \\
\hline
\end{tabular}

Figura 2. Matriz de probabilidades de transição

Deve-se interpretar cada linha da tabela da figura como as probabilidades de transição de um estado para os outros estados do sistema. Por exemplo, a primeira linha mostra o que normalmente ocorre a partir da tela inicial do curso de Computação após o login: $18 \%$ dos alunos vão para a sala de tutoria (estado 3), $29 \%$ vão para a sala de disciplina (estado 10) e $20 \%$ decidem sair do sistema sem requisitar qualquer serviço (estado 1). Como dito anteriormente, a transição para o estado 1 deve ser interpretada como a saída do usuário do sistema (Logoff) e o retorno do usuário ao mesmo após um período de tempo (Login). Assume-se que o tempo de permanência do aluno fora do sistema é zero, pois este trabalho só tem interesse em modelar o comportamento do usuário na plataforma.

É possível visualizar o diagrama de transições da cadeia de Markov na Figura 3. Não existe uma hierarquia bem definida nas transições entre os serviços do sistema de EAD. Pode-se, a partir de um estado qualquer da cadeia de Markov, alcançar praticamente todos os outros estados do modelo em um passo. 


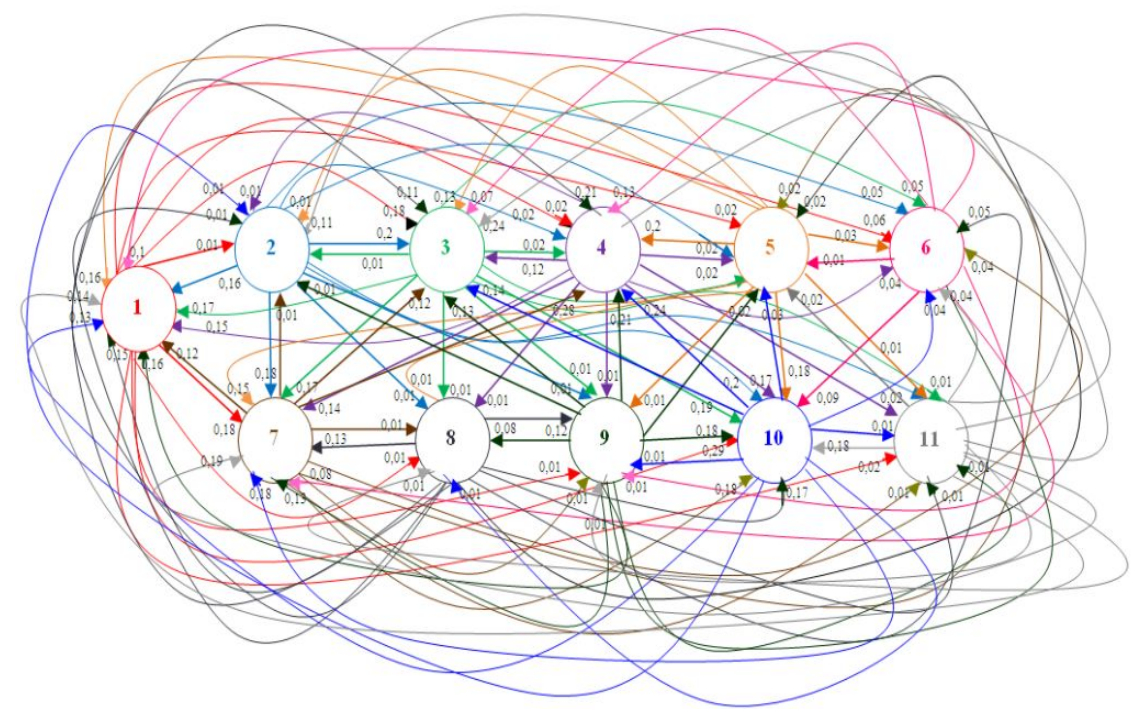

Figura 3. Diagrama de transições entre os estados do sistema

Embora esta característica facilite a navegação dos usuários pelos serviços do sistema, por outro lado, polui as interfaces do sistema que apresenta de uma vez quase todos os serviços oferecidos, mesmo aqueles poucos (ou nunca) utilizados, conforme Figura 4.

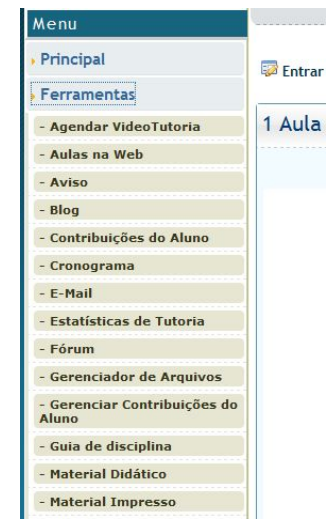

Figura 4. Ferramentas na Sala de Disciplina

Com a geração da matriz de probabilidades de transição, foi então possível calcular as probabilidades estacionárias do modelo mostradas na Figura 5: o aluno passa quase $20 \%$ do tempo na Sala de Disciplina e $18 \%$ do tempo fazendo upload/download de arquivos da plataforma. A ferramenta de e-mail é pouco utilizada pelos alunos (cerca de $8 \%$ do tempo gasto na plataforma). Considerando que um aluno utiliza o sistema por cerca de 90 minutos, isto significa que ele gasta por dia cerca de 18 minutos na Sala de Disciplina, 16 minutos com o gerenciador de arquivos e 7 minutos com os e-mails da plataforma. O pouco tempo no uso diário destas ferramentas pode ser explicado pelo fato do CEDERJ disponibilizar as vídeo-aulas do curso de Computação em DVDs. Assim, os alunos podem estudar as matérias das disciplinas sem precisar ficar conectado ao sistema de EAD. Esta suposição é confirmada pela análise dos logs do CEDERJ, pois o número de requisições e a duração das sessões dos usuários na plataforma aumentam significativamente nas duas semanas que antecedem às provas presenciais nos pólos. Neste período é que os alunos apresentam as dúvidas e iniciam uma participação mais ativa nos fóruns e salas de tutoria. 


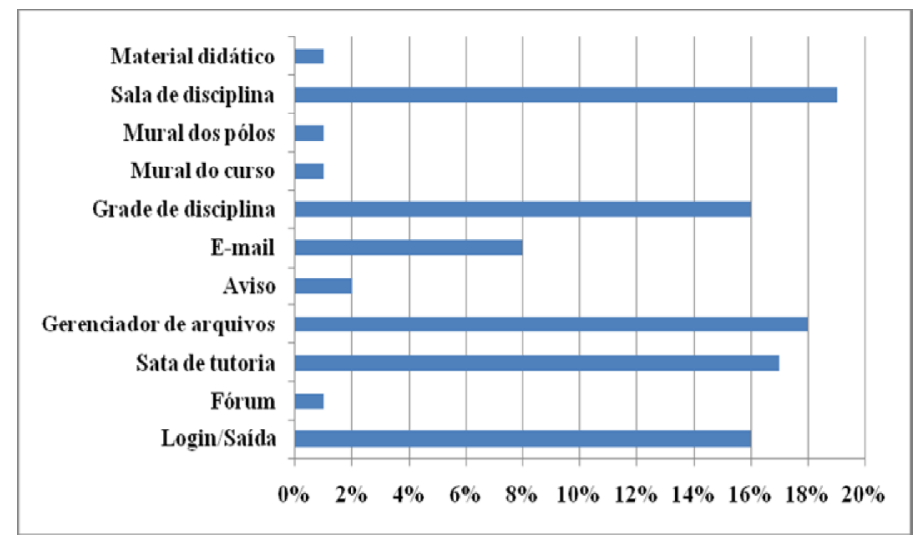

Figura 5. Probabilidades estacionárias do sistema

É interessante destacar que a ferramenta Sala de Disciplina pode ser acessada a partir de quase todas as interfaces do sistema como mostram as Figuras 6 e 7. Sendo que na inicial, o aluno visualiza a lista de todas as disciplinas em que ele se encontra matriculado. A ênfase dada a esta ferramenta pode ser facilmente explicada. Se um aluno quiser obter qualquer informação sobre provas, avisos, gabaritos, ele irá inicialmente à Sala da Disciplina.

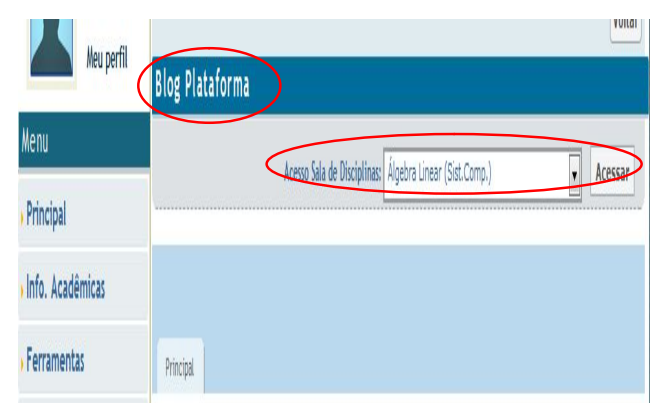

Figura 6. Acesso facilitado para Sala de Disciplina

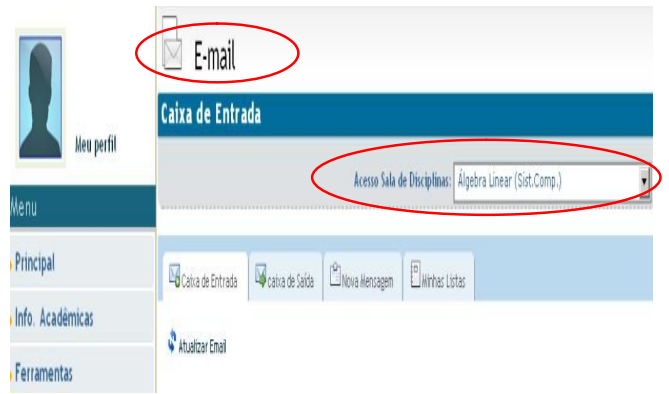

Figura 7. Acesso facilitado para Sala de Disciplina

A partir da Sala de Disciplina, o aluno tem a possibilidade de acessar algumas funções específicas (i.e.: aviso, sala de tutoria, material didático, etc.). Isto significa que determinados serviços do sistema só são disponibilizados após a escolha da disciplina. Isto faz sentido, pois assim se evita que o sistema precise pedir o nome da disciplina a cada requisição de serviço.

Cabe ressaltar que é possível identificar o caminho de navegação normalmente utilizado pelos alunos de Computação usando a matriz de probabilidades de transição (Figura 2). Um aluno que acessa o sistema, após a identificação (Login), vai para a sala de disciplina em $29 \%$ dos casos. A partir da sala de disciplina o aluno acessa o gerenciador de arquivos com uma freqüência de $24 \%$. Em seguida, o aluno pode voltar 
para a sala de disciplina (17\%) ou continuar no gerenciador de arquivos $(32 \%)$. Portanto, é possível dizer que o aluno faz uso de quatro ou cinco serviços a cada acesso ao sistema e que estes serviços normalmente são os mesmos.

Pode-se notar também que algumas funções possuem pouco acesso no sistema (Figura 5). Funções como fórum, aviso, e-mail, mural dos cursos, mural dos pólos e material didático não chegam a ter $10 \%$ do tempo em que os usuários ficam conectados à plataforma do CEDERJ. Além disso, existem funções que nunca são acessadas pelos usuários do sistema EAD. Infelizmente, o modelo markoviano não fornece indícios sobre a razão do pouco uso dessas ferramentas. Para se obter uma resposta a esta questão seria necessária uma pesquisa de opinião junto aos alunos dos CEDERJ.

\section{Análise dos Resultados}

Após a geração da matriz de probabilidades de transição entre estados, observou-se que a avaliação de usabilidade, baseando-se nos modelos markovianos, possibilita analisar cinco dos seis critérios de usabilidade especificados por Gassenferth et al (Gassenferth et al, 2008), apresentados na Seção 2.

O critério "Satisfação" não pode ser verificado com os modelos markovianos tendo em vista que o mesmo diz respeito à percepção dos usuários com o uso do sistema, o que seria possível com entrevistas ou aplicação de questionários. Através da utilização de modelos markovianos foi possível entender como algumas decisões de desenvolvimento implementadas podem influenciar o comportamento e a interação do usuário com o sistema, impactando diretamente na usabilidade do mesmo.

Baseando-se nos critérios possíveis de serem avaliados foi possível obter os seguintes resultados:

\subsection{Facilidade de Relembrar}

Através do diagrama de transição, não foi possível verificar onde o sistema auxilia o usuário no que diz respeito à facilidade de relembrar. Note que nos estados listados não existe nenhuma opção para guardar as informações ou ações executadas a fim de auxiliar o usuário no futuro. É importante observar que apesar do sistema dispor a seus usuários 81 ferramentas, nenhuma delas esta vinculada a este critério. Portanto, uma melhoria que pode ser sugerida é a definição de um histórico com as últimas atividades executadas pelos usuários no sistema;

\subsection{Facilidade de Aprender}

O sistema apresenta módulos que facilitam a compreensão dos usuários. Assim, para obter informações ou material didático de uma determinada disciplina, deve-se ir primeiro para a sala da disciplina desejada, onde o usuário encontra não só o que deseja como outras facilidades relacionadas à disciplina.

\subsection{Controle de Erros}

Ao analisar os diagramas com os estados do sistema verifica-se que não existe um estado para a análise e tratamento de erros. Atualmente, quando um erro ocorre, o aluno necessita enviar e-mail para o suporte da plataforma explicando o problema e aguardar um retorno. Uma possível solução seria uma ferramenta ou estado para tratamento dos erros integrado ao sistema. 


\subsection{Eficiência}

Com poucas exceções, é possível a partir de uma determinada ferramenta do sistema alcançar qualquer outra ferramenta. Portanto, o usuário não precisa perder tempo navegando entre menus diferentes realizando sua tarefa com maior produtividade e rapidez;

\subsection{Eficácia}

Embora o sistema avaliado forneça 81 diferentes ferramentas, apenas onze delas são usadas pelos alunos da Computação. Seria interessante então verificar se o comportamento dos alunos dos outros cursos é semelhante aos dos alunos do curso estudado. Uma possível solução para melhorar a avaliação deste critério é criar uma interface mais enxuta para o curso de Computação, apresentando apenas as ferramentas que realmente são utilizadas pelos alunos.

De acordo com os estados gerados para o sistema é possível saber com antecipação como ocorre à interação dos usuários com o sistema e suas devidas métricas de probabilidades, propiciando o desenvolvimento de uma interface com melhor usabilidade. Um usuário que se conecta hoje a plataforma CEDERJ irá utilizar com mais frequiência algumas ferramentas em detrimento a outras. Essa informação auxilia na melhor avaliação do que melhorar no sistema a fim de que o mesmo realize as tarefas a que foi submetido com um menor nível de interação com o usuário.

\section{Considerações Finais}

Para avaliar a usabilidade é possível aplicar diferentes métodos de avaliação. Um deles é por meio de aplicação de modelos markovianos, que possibilitam fornecer comparações quantitativas sobre usabilidade do projeto. Assim, a presente pesquisa teve por objetivo investigar a utilização de modelos markovianos na avaliação de usabilidade, tendo como estudo de caso um sistema EAD, de forma a contribuir para a tomada de decisões relacionadas ao projeto, auxiliando novas propostas de melhorias a serem implementadas em sistemas deste tipo.

Esse estudo possibilitou a geração de uma matriz de probabilidades entre estados e ações, que foi utilizada na avaliação de usabilidade utilizando os critérios especificados por Gassenferth et al (2008); escolheu-se estes critérios por ter sido criados com base em um estudo qualitativo e comparativo dos métodos utilizados para avaliação da usabilidade de autores conceituados da área de IHC. Observou-se que cinco critérios (de um total de seis) podem ser avaliados com os modelos markovianos: facilidade de relembrar, facilidade de aprender, controle de erros, eficiência e eficácia.

O estudo mostrou que com a utilização de modelos de Markov é possível identificar necessidades de melhorias na usabilidade do sistema com base nas métricas utilizadas pelos autores mais conceituados da área. Os resultados obtidos mostraram que é possível melhorar a usabilidade do sistema de EAD do CEDERJ com a introdução de algumas mudanças como, por exemplo: a definição de um módulo para identificar e reportar erros do sistema; a definição de um histórico com as ações executadas pelos usuários; e a definição de uma interface mais simples onde estivesse presente apenas as ferramentas mais utilizadas pelos alunos. 
Além disso, foi possível constatar com este estudo a pouca interatividade existente entre os alunos do curso. Uma prova disso é a baixa utilização da ferramenta de e-mails. Uma sugestão para resolver este problema é a criação de uma rede social na plataforma para alunos, tutores e professores. Este tipo de ferramenta já mostrou, em diversas situações, que é adequada para a integração e a motivação de pessoas.

Como trabalhos futuros pretende-se utilizar os resultados dos modelos de markov na avaliação de demais critérios que influenciem na usabilidade do sistema, como por exemplo a taxonomia apresentada por Ferreira e Nunes (2008).

\section{Referencias}

Bastien, J. M. C.; Scapin, D. L. (1993). Human factors criteria, principles, and recommendations for HCI: methodological and standardization issues. França: INRIA.

Booch, G. (1994). Object Oriented Analysis and Design with Applications (2a ed.). California, AddisonWesley.

Cunha, U. S.; Machado, S. A. ; Figueiredo Filho, A.; Sanquetta, C. R. (2010). Predição da estrutura diamétrica de espécies comerciais de terra firme da Amazônia por meio de matriz de transição. Ciência Florestal, Santa Maria, v. 12, n. 1, p. 109-122.

CEDERJ (2011). Acessado em Fevereiro (2011), http://portal.cederj.edu.br/fundacao/.

Ferreira, S. B. L, Nunes, R. R. (2008). e-Usabilidade. Rio de Janeiro, LTC Editora.

Gassenferth, W.; Santos, R. C.; Machado, M. A. S. (2008). Systems usability evaluation metrics review. Global Business and Technology Association Conference - GBATA, Systems Usability Evaluation Metrics Review, Madri, Espanha

Jordan, P. W. (1998). An Introduction to Usability. London, UK: Taylor e Francis.

Kleinrock, L. (1975). Queueing Systems - Theory, Vol. I. Wiley-Interscience.

Kitajima, M; Kariya, N., Takagi, H. and Zhang, Y. (2005). Evaluation of website usability using Markov chains and latent semantic analysis. IEICE Transactions on Communications. E88B (4),1467-1475.

Marinho, A.; Cardoso, S. S.; Almeida, V. V. (2010). Disparidades nas filas para transplantes de órgãos nos estados brasileiros. Cad. Saúde Pública [online]. vol.26, n.4, pp. 786-796. ISSN 0102-311X.

Morais, I. A. C.; Bertoldi, A.; Anjos, A. T. M. (2010). Estimativa de um modelo não linear para as exportações brasileiras de borracha no período 1992-2006. Rev. Econ. Sociol. Rural [online]. vol.48, n.3, pp. 679-704. ISSN 0103-2003.

Nielsen, J., Loranger, H. (2007). Usabilidade na web: projetando websites com qualidade (1a ed.). Rio de Janeiro: Elsevier.

Prates, R. O., Barbosa, S. D. J. (2003). Avaliação de Interfaces de Usuário - Conceitos e Métodos. Jornada de Atualização em Informática (JAI) do XXIII Congresso da SBC, v. 2, p. 245-293.

Preece, J., Rogers, Y., Sharp, H. (2005). Design de Interação: além da interação homem-computador (1a ed). Porto Alegre: Bookman.

Quesenbery, W. (2001). What does usability mean: Looking beyond 'ease of use'. In: 48th Annual Conference Society for Technical Communication. Chicago.

Rocha, H.; Baranauska, M. (2000) Design e avaliação de interfaces humano-computador. Instituto de Computação.

Shackel, B. (1986). Ergonomics in design for usability. In: HCI 86 Conference on People and Computer. New York: Cambridge University Press.

Shneiderman, B. (1998). Designing the User Interface: Strategies for Effective Human-Computer Interaction. 3. ed. EUA: Addison Wesley.

Sommerville, I. (2007) Engenharia de Software, 8a ed., Addison-Wesley.

Thimbleby, H.; Cairns, P.; Jones, M.(2001). Usability analysis with markov models. ACM Trans. Comput. Human Inter. 8, 2, 99-132. 